\title{
Wnt ligands $3 a$ and $5 a$ regulate proliferation and migration in human fetal liver progenitor cells
}

\author{
Zhiwen Liu, Vijay Kumar Kuna, Bo Xu, Suchitra Sumitran-Holgersson \\ Laboratory for Transplantation and Regenerative Medicine, Institute of Clinical Sciences, Sahlgrenska Academy, University of Gothenburg, \\ Gothenburg, Sweden \\ Contributions: (I) Conception and design: Z Liu, S Sumitran-Holgersson; (II) Administrative support: S Sumitran-Holgersson; (III) Provision of study \\ materials or patients: S Sumitran-Holgersson; (IV) Collection and assembly of data: Z Liu, B Xu; (V) Data analysis and writing: All authors; (VI) \\ Manuscript writing: All authors; (VII) Final approval of manuscript: All authors. \\ Correspondence to: Prof. Suchitra Sumitran-Holgersson. Laboratory for Transplantation and Regenerative Medicine, Sahlgrenska Science Park, \\ Medicinaregatan 8A, $2^{\text {nd }}$ Floor, S-413 46 Gothenburg, Sweden. Email: suchitra.holgersson@gu.se.
}

Background: Since human fetal liver progenitor cells (hFLPC) can differentiate into multiple liver cell types in vitro and in vivo, hFLPC may be a suitable source for cell therapy and regeneration strategies. Imperative for effective clinical applications of hFLPC is the enhanced knowledge of growth factors that mediate and improve migration and proliferation. The canonical wingless/int-1 (Wnt) signal transduction pathway is known to play a key role in proliferation and migration of stem cells. So, we investigated a role for Wnt3a and Wnt5a ligands in regulating the proliferation and migration of hFLPC.

Methods: We used alamarBlue assay and transwell migration assay and examined proliferation and migration of hFLPC to Wnt3a and Wnt5a. In addition, the target genes of Wnt signal transduction pathway was identified using microarray analysis and validated by quantitative real-time polymerase chain reaction (qPCR).

Results: We found that Wnt3a or Wnt5a independently significantly increased migration and proliferation in a dose-dependent manner which was significantly inhibited by Wnt inhibitors Wnt-C59 or KN-62. Addition of Wnt3a to hFLPC resulted in increased mRNA expression of the known Wnt target genes Axin-2, DKK2, while Wnt5a increased CXCR7, all of which are closely associated with an enhanced proliferation capacity of stem cells.

Conclusions: Thus, we report that Wnt3a and Wnt5a may play an important role in the proliferation and migration of hFLPC by possibly regulating key target genes-involved in these processes. Incorporating recombinant human $\mathrm{Wnt} 3 \mathrm{a}$ and $\mathrm{Wnt} 5 \mathrm{a}$ in regenerative strategies using liver stem/progenitor cells might improve the process of liver regeneration.

Keywords: Wnt3a; Wnt5a; Axin2; DKK2; CXCR7; regenerative medicine

Received: 17 October 2019; Accepted: 18 January 2020; Published: 25 October 2021.

doi: $10.21037 / \operatorname{tgh} .2020 .01 .12$

View this article at: http://dx.doi.org/10.21037/tgh.2020.01.12

\section{Introduction}

Liver disease is a leading cause of morbidity and mortality worldwide (1). Liver diseases are recognized as the second leading cause of mortality amongst all digestive diseases in the US (2). Global prevalence of cirrhosis from autopsy studies ranges from $4.5 \%$ to $9.5 \%$ of the general population (3).
Hence, it is estimated that more than fifty million people in the world, taking the adult population, would be affected with chronic liver disease. While allogeneic liver transplantation is the only definitive treatment for the growing number of patients with end-stage liver disease, few patients on the organ waiting list undergo transplantation, given the limited availability of donor organs. As a result, there is a need to 
find strategies that could serve as temporary or definitive alternatives to liver transplantation.

Cell-based technologies of regenerative medicine using autologous or allogeneic hepatocytes or stem/progenitor cells capable of differentiation into liver cells offer a novel approach to better management and diagnostics of liver disease (4-6). In addition, human hepatocytes are used in extracorporeal liver support systems and their long-term cultures are gradually substituting animal experiments in drug testing and in vitro disease modeling. Another promising regenerative approach to increase the donor organ pool is to use allogeneic or xenogeneic decellularized livers as a scaffold to engineer functional liver tissue with cells ex vivo (7). Decellularization of mouse, rat, goat, sheep, pig, non-human primate and human liver tissue has been accomplished with several detergent-based approaches as well as with freeze-thaw cycles, and resulted in threedimensional (3D) acellular scaffolds that are generally devoid of detectable residual DNA and nuclei. The decellularized liver scaffolds produced by different methods generally retain major extracellular matrix (ECM) proteins (8-10). Repopulation of decellularized livers has been reported using a number of different cell types, including transformed cell lines such as HEPG2, fetal liver cells, endothelial cells, embryonic stem cells, induced pluripotent stem cells, mesenchymal stem cells (11-14). However, so far only partial recellularization of liver tissue has been achieved. One potential approach to improve recellularization of decellularized liver scaffolds is to use cells with high migratory and proliferative capacity.

Canonical wingless/int-1 (Wnt) signaling controlled by its effector $\beta$-catenin is known to play an important role in liver regeneration and liver cell proliferation (15-17). Wnt signaling also has a prominent role in stem cell biology, including self-renewal, pluripotency, and differentiation of both embryonic stem and somatic stem cells (18). In fact, an active Wnt signaling has been found during progenitor cellmediated regeneration of the liver (19-21). To date most of this work has been performed using rodent liver progenitor cells, but not human liver progenitor cells. Furthermore, the role of Wnt signaling in migratory capacity of human fetal liver progenitor cells (hFLPC) has not been studied.

Therefore, we investigated a role for $\mathrm{Wnt} 3 \mathrm{a}$ and $\mathrm{Wnt} 5 \mathrm{a}$ signalling in regulating the proliferation and migration of hFLPC.

\section{Methods}

\section{Cells and cell cultures}

Three human fetal liver tissues were obtained from aborted fetuses at 9-10 weeks of gestation in accordance with ethical permission from Regionala etikprövningsnämden i Göteborg. Gestational age is given as menstrual age. The fetuses used were from pregnancies with no apparent abnormalities and no fetuses with anomalies were included. Fetal liver was dissected and placed in a sterile tube containing roswell park memorial institute (RPMI) 1640 medium (Gibco, Invitrogen Corp., UK). The liver was then disintegrated into a single cell suspension by passage through a $70-\mu \mathrm{m}$ nylon mesh. The single cell suspension was centrifuged at $200 \times \mathrm{g}$ for $10 \mathrm{~min}$ to pellet the cells. Thereafter cell samples were kept frozen in liquid nitrogen until further analyses.

The primary hFLPC were seeded on human placenta collagen coated culture flasks and cultivated in Dulbecco modified eagle medium (DMEM, Lonza, Belgium) containing $10 \%$ heat inactivated human $\mathrm{AB}$ serum (Sigma, USA), 1\% L-glutamine (Gibco, UK) and $1 \%$ penicillin streptomycin (Gibco, USA). After 2 days the medium was replaced to remove all non-adherent cells. One percent sodium pyruvate (Gibco, China), 1\% minimum essential media (MEM, Gibco, USA), hepatocyte growth factor (HGF, $30 \mathrm{ng} / \mathrm{mL}$ ), epidermal growth factor (EGF, $20 \mathrm{ng} / \mathrm{mL}$ ), basic fibroblast growth factor (bFGF, $10 \mathrm{ng} / \mathrm{mL}$ ), vascular endothelial growth factor (VEGF, $5 \mathrm{ng} / \mathrm{mL}$ ), interleukin-6 (IL-6, $2 \mathrm{ng} / \mathrm{mL}$ ), and fibroblast growth factor 9 (FGF-9, $10 \mathrm{ng} / \mathrm{mL}$ ) (all from PeproTech, USA) were added to the above medium and the medium was replaced every third day.

In addition to primary hFLPC, simian virus 40 large $\mathrm{T}$ antigen transfected human fetal liver cells (SV40LT-HFL) established by our lab (22) were used for comparison in all experiments.

\section{Flow cytometric analysis}

Primary hFLPC ( $n=3$ ) (passages 3 to 4) were analyzed by flow cytometry for expression of liver cell markers using antibodies to cytokeratins (CK) 8 (ab9023, Abcam, UK), CK18 (SC51583, Santa Cruz, USA), cytochrome P450 3A7 (CYP3A7, C4993, Sigma, USA), albumin (A6684, Sigma, USA) and alpha fetoprotein (AFP, SC-8399, Santa Cruz, USA). Cells stained with fluorescein isothiocyanate conjugated goat antimouse secondary antibody (F0257, Sigma, USA) only were used as negative control. Briefly, $0.5 \times 10^{5}$ cells were washed twice with phosphate buffer saline (PBS) and permeabilized with $0.5 \%$ saponin. Primary antibodies were added and incubated at $4{ }^{\circ} \mathrm{C}$ on ice for $30 \mathrm{~min}$. After washing twice with PBS, cells were stained by secondary antibody at $4{ }^{\circ} \mathrm{C}$ on ice for $30 \mathrm{~min}$. Finally, cells were washed with PBS twice 
and then analyzed on a Becton Dickinson flow cytometer (BD Biosciences, Accuri C6, USA). The percentage of cells expressing the marker was noted from histogram overlay plots and gating the cells. The mean percentage of cells expressing the marker was calculated and presented.

\section{Cell migration assay}

The migration of SV40LT-HFL cells and hFLPC $(n=1)$ were assayed using 24-well transwell chambers (Costar, USA) fitted with $8-\mu \mathrm{m}$ pores filter inserts. For determination of chemotaxis, the filter inserts were coated on both sides with $10 \mu \mathrm{g} / \mathrm{mL}$ of collagen type IV (Advanced BioMatrix, USA) and 12.5 or 25 or 50 or 100 or $200 \mathrm{ng} / \mathrm{mL}$ of Wnt 3 a or Wnt5a diluted in $600 \mu \mathrm{L}$ of serum-free DMEM were placed below the inserts of transwell chambers, while $100 \mu \mathrm{L}$ of serum-free DMEM containing 50,000 cells was placed on the upper side of the inserts and incubated for 24 hours at $37^{\circ} \mathrm{C}$. At the end of assays, the inserts were detached from the plastic support, fixed in methanol, the non-migrated cells from the upper side of the inserts were wipe off with cotton swabs, and stained with Giemsa stain. Cells that have migrated through the pores to the lower side of the inserts were observed under an inverted light microscope. The filter inserts were visualized using a $20 \times$ objective, and the number of cells that had migrated across the filters was counted for all filters in 5 random fields and the average was represented. Unpaired two-tailed Student's $t$-test was used to compare differences between all treatment groups.

\section{Cell proliferation assay}

Cell proliferation was evaluated using the alamarBlue assay as specified by the manufacturer (Invitrogen, USA). To perform proliferation assay, SV40LT-HFL cells and hFLPC $(n=1)$ were plated at a density of 4,000 cells/well in 96-well microplates and 2\% human serum DMEM media with or without Wnt3a or Wnt5a was added at 50 or 100 or $200 \mathrm{ng} / \mathrm{mL}$. To test whether addition of Wnt inhibitors to cell culture also inhibits cell proliferation, Wnt inhibitor C59 (Selleckchem, Germany) and calmodulin-dependent protein kinase II (CamKII) inhibitor KN-62 (Selleckchem, Germany) at $10 \mu M$ were added in same media with Wnt3a and Wnt5a individually. C59 is a small molecule Wnt inhibitor that inhibits both canonical and non-canonical Wnt signaling pathways while $\mathrm{KN}-62$ is a selective CamKII inhibitor that was suggested to activate non-canonical pathway in presence of Wnt5a $(23,24)$. After 48 hours, alamarBlue at $10 \% \mathrm{v} / \mathrm{v}$ was added and incubated for 4 hours. Later, fluorescence was measured with microplate reader (Synergy2, BioTek, USA) with excitation and emission at 530 and $590 \mathrm{~nm}$, respectively. All experiments were run in multiples of 5. Unpaired two-tailed Student's $t$-test was used to compare differences between all treatment groups.

\section{Gene expression microarray analysis*}

Human Gene 2.1 ST Arrays interrogating the expression of 53,617 transcripts, including 27,384 annotated coding transcripts were used for analysis of global gene expression profiling. Target synthesis and hybridizations were performed at the Bioinformatic and Expression Analysis core facility (BEA, www.bea.ki.se, Novum, Karolinska Institutet, Huddinge, Sweden) according to standard protocols. Signal summarization, normalization and background correction were performed using the Robust Multichip Analysis in Affymetrix Expression Console. Unpaired two-tailed Student's $t$-tests were used to compare sample groups. We applied a filter of $\mathrm{P}<0.05$ for significantly modulated gene expression and at least a 1.5 -fold change in mean differential expression. Gene ontology analysis and identification of enriched pathways were performed using the database for annotation, visualization and integrated discovery bioinformatics resource 6.7 .

\section{$R N A$ extraction and quantitative real-time polymerase chain reaction ( $q P C R$ )}

SV40LT-HFL cells and hFLPC $(\mathrm{n}=3)$ were cultured in 6-well cell culture plates in $10 \%$ human $\mathrm{AB}$ serum containing DMEM media. After 18 hours incubation, the medium was changed to DMEM containing $1 \%$ human $\mathrm{AB}$ serum for 6 hours followed by treatment with $\mathrm{Wnt} 3 \mathrm{a}$ or Wnt5a at $100 \mathrm{ng} / \mathrm{mL}$ (R\&D Systems, UK) for 12 hours in triplicates. Total RNA was extracted using RNeasy Mini Kit (Qiagen, USA). Complementary DNA was synthesized using TaqMan reverse transcription kit (Applied Biosystems, USA). qPCR was performed in a 7500 Fast

\footnotetext{
*The microarray data generated during the current study is available in link https://www.ncbi.nlm.nih.gov/geo/query/acc.cgi?acc=GSE106755.
} 
Real-Time PCR (Applied Biosystems, USA) with fast synergy brand green master mix (Applied Biosystems, USA) according to conditions specified by the manufacturer. Gene expression levels were normalized to $36 \mathrm{~B} 4$ in primary hFLPC and glyceraldehyde 3-phosphate dehydrogenase in SV40LT-HFL cells. The specificity of all primer pairs was

Table 1 Primer sequences

\begin{tabular}{|c|c|c|}
\hline No. & Gene & Sequence \\
\hline \multirow[t]{2}{*}{1} & Axin-2 & F: TGGCAACTCAGTAACAGCCC \\
\hline & & R: GCTCATCTGAACCTCCTCTCTT \\
\hline \multirow[t]{2}{*}{2} & $D K K 2$ & F: CCCAGTACCCGCTGCAATAA \\
\hline & & R: CGATCTCTGTGCCGAGTACC \\
\hline \multirow[t]{2}{*}{3} & CXCR7 & F: CAGCAGAGCTCACAGTTGTTG \\
\hline & & R: GAGGCGGGCAATCAAATGAC \\
\hline \multirow[t]{2}{*}{4} & $36 B 4$ & F: GTGTTCGACAATGGCAGCAT \\
\hline & & R: GACACCCTCCAGGAAGCGA \\
\hline \multirow[t]{2}{*}{5} & $G A P D H$ & F: GACCCCTTCATTGACCTCAACT \\
\hline & & R: GAATTTGCCATGGGTGGAAT \\
\hline
\end{tabular}

checked with melting curve analysis. Primer sequences were provided in Table 1. For qPCR analysis, unpaired two-tailed Student's $t$-test was used to compare differences between two parallel treatment groups.

\section{Results}

\section{Characterization of bFLPC}

Hepatic cell markers were detected in all hFLPC tested using flow cytometry. The hFLPC showed expression of CK8, CK18, CYP3A7, AFP and albumin and the mean percentage of cells expressing the markers were $53 \% \pm 21 \%$, $59 \% \pm 19 \%, 73 \% \pm 9 \%, 61 \% \pm 22 \%$ and $60 \% \pm 11 \%$ respectively (Figure 1).

\section{Wnt ligands $3 a$ and $5 a$ induced migration of SV4OLT-HFL cells and bFLPC}

Wnt3a and Wnt5a induced cell migration in a dosedependent manner up to $100 \mathrm{ng} / \mathrm{mL}$ in both SV40LT-HFL cells and hFLPC (Figure 2A,2B). Further increasing the concentration to $200 \mathrm{ng} / \mathrm{mL}$ for both Wnt3a and Wnt5a, SV40LT-HFL cells did not show increased number of


\begin{tabular}{|c|c|}
\hline Marker & $\begin{array}{l}\text { Mean percentage of cells } \\
\text { expressing the marker }\end{array}$ \\
\hline CK8 & $53 \% \pm 21 \%$ \\
\hline CK18 & $59 \% \pm 19 \%$ \\
\hline Albumin & $73 \% \pm 9 \%$ \\
\hline CYP3A7 & $61 \% \pm 22 \%$ \\
\hline AFP & $60 \% \pm 11 \%$ \\
\hline
\end{tabular}

Figure 1 Characterization of primary hFLPC. Primary hFLPC characterized by flow cytometry using antibodies to liver markers showed positive staining for CK8, CK18, the P450 enzyme CYP3A7, AFP and albumin. hFLPC, human fetal liver progenitor cells; AFP, alpha fetoprotein. 
A

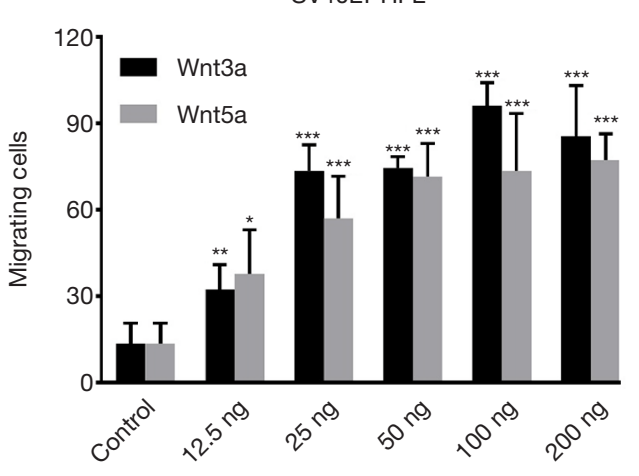

C

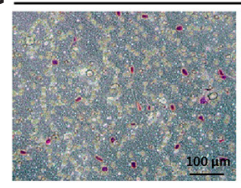

Control
SV40LT-HFL

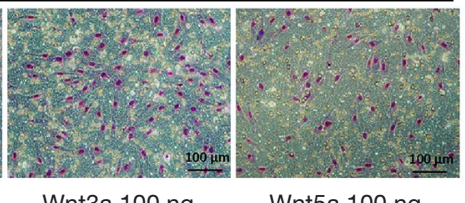

B



$\mathrm{D}$

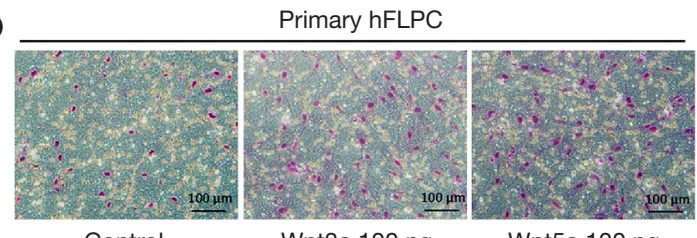

Figure 2 Wnt ligands 3a and 5a induced migration of SV40LT-HFL cells and primary hFLPC. The representative microscopic pictures of transwell after Giemsa staining showing high number of migrated cells to Wnt3a and Wnt5a at $100 \mathrm{ng}$ concentration compared to control for SV40LT-HFL cells (C) and primary hFLPC (D). The quantification of migrated cells showed increased number of cells migrated to Wnt3a and Wnt5a in dose dependent manner as compared to controls for both SV40LT-HFL cells (A) and primary hFLPC (B). Each bar represents the mean value of the number of migrated cells \pm SD of 5 random field determinations. Magnification: 200x. *, $\mathrm{P}<0.05 ;{ }^{* *}$, $\mathrm{P}<0.01$; ${ }^{* * *}, \mathrm{P}<0.001$ vs. control). hFLPC, human fetal liver progenitor cells; SV40LT-HFL, simian virus 40 large $\mathrm{T}$ antigen transfected human fetal liver cells; SD, standard deviation.

migrating cells while hFLPC show a decrease in number of migrating cells only for Wnt5a but did not show any change for Wnt3a. The representative microscopic pictures from Giemsa staining of inserts showing high number of SV40LT-HFL cells and hFLPC migration towards Wnt3a and Wnt5a as compared to controls (Figure $2 C$ and Figure $2 D$ respectively).

\section{Wnt ligands $3 a$ and 5 a stimulated proliferation of SV4OLT-HFL cells and bFLPC}

Addition of Wnt3a or Wnt5a to cell culture induced proliferation consistently in both SV40LT-HFL cells and hFLPC (Figure $3 A, 3 B$ ) as seen from alamarBlue assay.

\section{Wnt-C59 and $\mathrm{KN}-62$ inbibit Wnt ligands $3 a$ and 5 a-induced cell proliferation}

As shown in (Figure $3 C-3 F$ ), C59 and $\mathrm{KN}-62$ at $10 \mu \mathrm{M}$ inhibited both Wnt3a and Wnt5a induced proliferation in SV40LH-HFL cells and hFLPC.

\section{Gene ontology analysis}

Assigning the Wnt3a-upregulated genes in SV40LTHFL cells to functional categories, we found that genes associated with Wnt receptor signaling pathway, embryonic morphogenesis, positive regulation of cell differentiation, positive regulation of cell proliferation, positive regulation of signal transduction and regulation of apoptosis were highly enriched (Figure 4A). This is consistent with proliferation induced by Wnt3a in SV40LT-HFL cells (Figure 3A). For Wnt5a-upregulated genes in SV40LTHFL cells, mitochondrion organization, neutral amino acid transport, serine family amino acid metabolic process, cofactor metabolic process, cellular amino acid biosynthetic process, response to testosterone stimulus, iron-sulfur cluster assembly, metallo-sulfur cluster assembly and response to cytokine stimulus were highly enriched (Figure $4 B$ ). In hFLPC, the main biological processes affected by Wnt3a were negative regulation of signal transduction, negative regulation of cell communication, sensory perception of smell and sensory perception of chemical stimulus, cell surface 


$$
\text { A }
$$

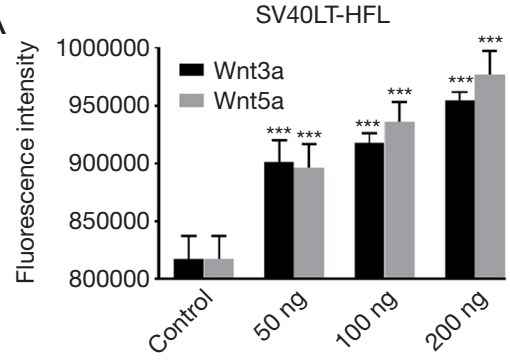

D

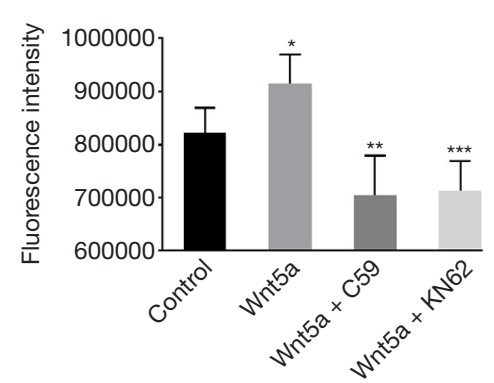

B

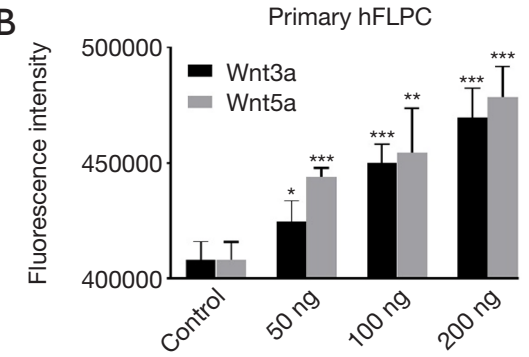

$E$

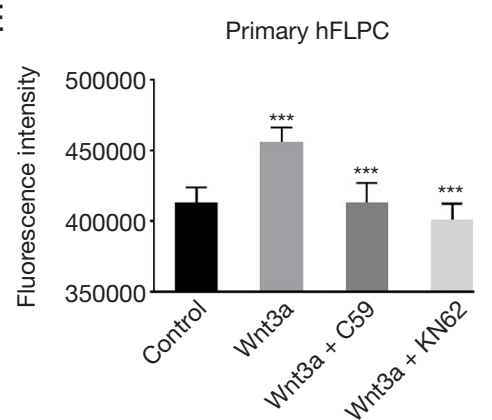

C



$\mathrm{F}$

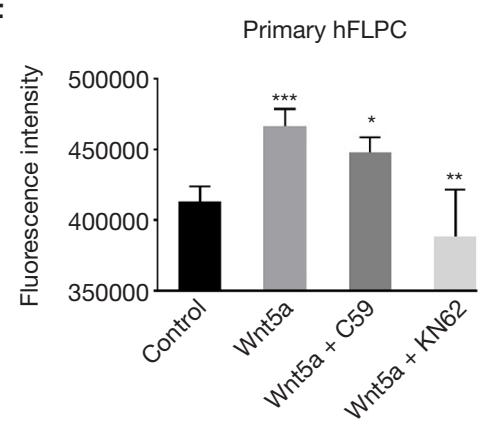

Figure 3 Wnt inhibitors (C59 and KN-62) inhibit Wnt3a and Wnt5a induced proliferation of SV40LT-HFL cells and primary hFLPC. Cell proliferation determined by alamarBlue assay showing induced proliferation by Wnt3a and Wnt5a in SV40LT-HFL cells (A) and primary hFLPC (B) in dose dependent manner. *, $\mathrm{P}<0.05$; **, $\mathrm{P}<0.01$; ${ }^{* *}, \mathrm{P}<0.001$ vs. control. Wnt inhibitors $\mathrm{C} 59$ and $\mathrm{KN}-62$ addition inhibited Wnt3a and Wnt5a induced cell proliferation in SV40LT-HFL cells (C and D respectively) and primary hFLPC (E and F respectively). The fluorescence intensity corresponds to the number of cells, thus more intensity corresponds to many cells in well and vice-versa. *, $\mathrm{P}<0.05$; **, $\mathrm{P}<0.01$; **, $\mathrm{P}<0.001$ vs. Wnt3a and/or Wnt5a group. The data shown are the average of five replicates \pm SD. hFLPC, human fetal liver progenitor cells; SV40LT-HFL, simian virus 40 large T antigen transfected human fetal liver cells; SD, standard deviation.

receptor linked signal transduction, sensory perception, neurobiological system process, smooth muscle cell differentiation, cognition and G-protein coupled receptor protein signaling pathway (Figure 4C), while G-protein coupled receptor protein signaling pathway, cell surface receptor linked signal transduction and cell-cell signaling were mainly affected by Wnt5a (Figure 4D).

\section{Validation of microarray results using $q P C R$}

We identified in microarray analysis Axin-2 and Dickkopf wnt signaling pathway inhibitor 2 (DKK2) as interesting target genes in response to $\mathrm{Wnt} 3 \mathrm{a}$ treatment and chemokine (C-X-C motif) receptor 7 (CXCR7) as interesting target gene in response to Wnt5a treatment in both primary hFLPC and SV40LT-HFL cells. The qPCR data also showed that expression of Axin-2 and DKK2 was substantially elevated by Wnt3a treatment in both SV40LT-HFL cells and hFLPC but not by Wnt5a (Figure $5 A$ and Figure $5 B$ respectively).
The expression of CXCR7 was upregulated by Wnt5a only in one out of three hFLPCs tested (Figure 5C). The three genes chosen were based on their known knowledge to promote proliferation and invasion in different cancer cells $(25,26)$, suggesting they might be the key genes regulated by Wnt signaling and then promote cell proliferation and migration seen in the primary hFLPC.

\section{Discussion}

Combining tissue specific stem/progenitor cells with the appropriate decellularized liver scaffolds to create functional livers is a promising technology. However, growth factors which play key roles in the essential processes of tissue regeneration may not remain within the matrix postdecellularization at critical concentrations required for liver regeneration. In order to improve this process, addition of exogenous growth factors that increase the migratory, proliferative and differentiation capacity of the seeded 
A Overrepresented gene ontology biologic processes for Wnt3a upregulated genes in SV40LT-HFL cells

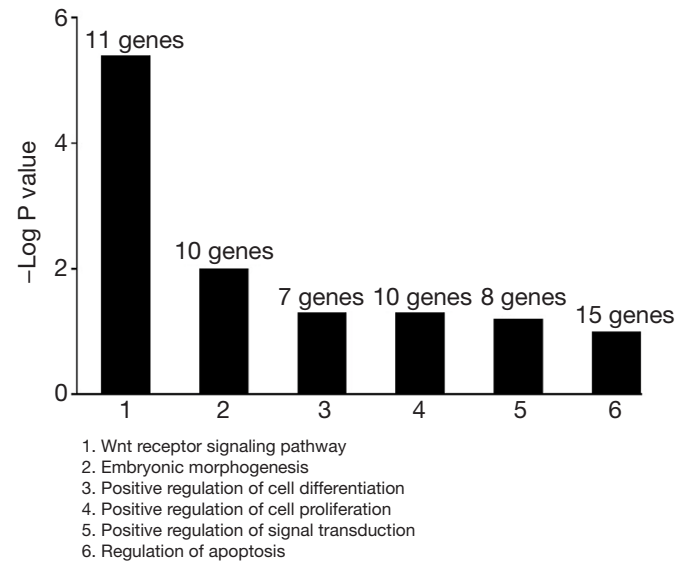

C Overrepresented gene ontology biologic processes for Wnt3a upregulated genes in primary hFLPC



B Overrepresented gene ontology biologic processes for Wnt5a upregulated genes in SV40LT-HFL cells



Overrepresented gene ontology biologic processes for Wnt5a upregulated genes in primary hFLPC

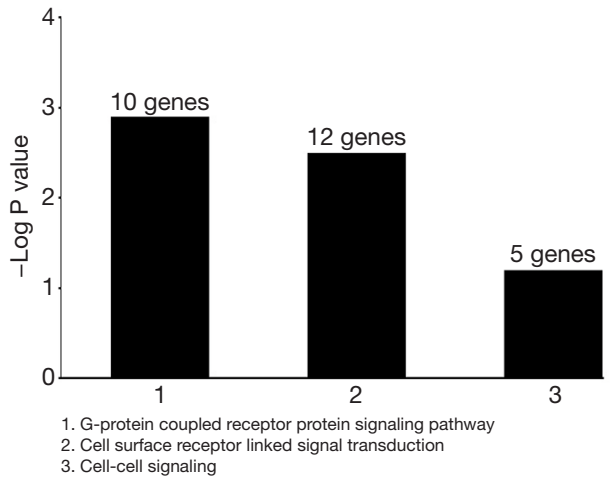

Figure $4 \mathrm{Wnt} 3 \mathrm{a}$ and Wnt5a regulate gene expression of SV40LT-HFL and primary hFLPC. Gene ontology (GO)-term enrichment analysis for Wnt3a-upregulated genes in SV40LT-HFL cells (A), Wnt5a-upregulated genes in SV40LT-HFL cells (B), Wnt3a-upregulated genes in primary hFLPC (C) and Wnt5a-upregulated genes in primary hFLPC (D) using Fisher's exact test through the DAVID bioinformatics resource 6.7. hFLPC, human fetal liver progenitor cells; SV40LT-HFL, simian virus 40 large $\mathrm{T}$ antigen transfected human fetal liver cells.

cells might be required. Here, we analyzed the effect of Wnt3a and Wnt5a on hFLPC, because these ligands are known to activate canonical and non-canonical Wnt signaling in various cell types (15-19). Our in vitro analysis by gene expression, proliferation and migration assays showed positive response after addition of Wnt3a and Wnt5a ligands in all processes. But we have not observed any significant differences between primary hFLPC and SV40LT-HFL cells.
For whole-liver recellularization, successful seeding of hepatocytes into a decellularized ECM and subsequent heterotopic transplantation of the graft has been reported in a rat model (27). However, distinct areas of low-density and poorly organized hepatocytes were discernable in the grafts and their morphology and function were not comparable to the native liver. Such problems usually result from seeded cells being non-migratory or proliferative and from lack of potential growth factors in decellularized matrix, 
A

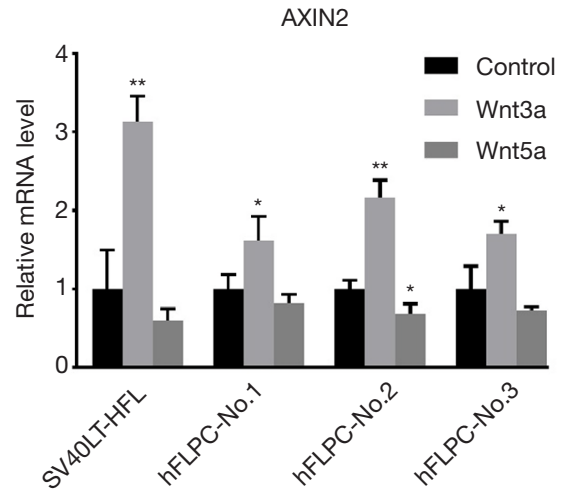

C

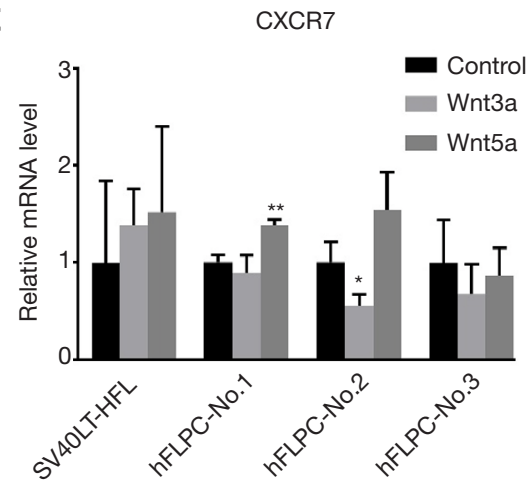

B

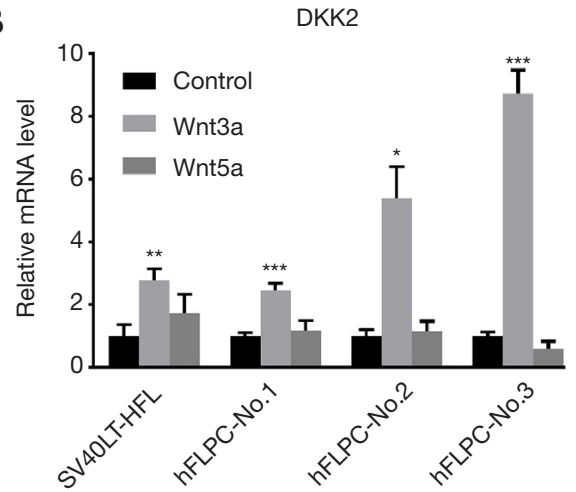

Figure 5 Validation of Wnt3a and Wnt5a upregulated genes in SV40LT-HFL and primary hFLPC using qPCR. Levels of mRNA for Axin-2 (A), DKK2 (B) and CXCR7 (C) were determined by qPCR after treatment with Wnt3a or Wnt5a for 18 hours in both SV40LTHFL cells and primary hFLPC. Wnt3a treatment increased Axin-2 and DKK2 expression in both SV40LT-HFL cells and primary hFLPC while Wnt5a showed increased expression of CXCR7 only in 1 out of 3 primary hFLPC tested. Data are shown as means with SD $(\mathrm{n}=3)$. *, $\mathrm{P}<0.05 ;{ }^{* *}, \mathrm{P}<0.01 ;{ }^{* * *}, \mathrm{P}<0.001$ compared with control group. hFLPC, human fetal liver progenitor cells; SV40LT-HFL, simian virus 40 large $\mathrm{T}$ antigen transfected human fetal liver cells; SD, standard deviation; qPCR, quantitative real-time polymerase chain reaction.

e.g., Wnt3a and Wnt5a. Since, it is reported that Wnt is a potent mitogen for hepatocytes and liver regeneration (28), we speculate $\mathrm{Wnt} 3 \mathrm{a}$ and $\mathrm{Wnt} 5 \mathrm{a}$ might be promising factors for supporting proliferation and migration of hFLPC in tissue engineering of human livers.

Even till today transplantation of at least functionally active hepatocytes is a challenge because of their tough in vitro maintenance, it is believed that decellularized livers will solve this problem by transplanting functional livers as they hold the desired growth factors in spatial and conformational way. To confirm that the decellularized matrix is suitable for supporting functional hepatocytes, the level of critical growth factors in the ECM, is an important parameter that should be taken into consideration (29,30). The presence of these growth factors stems from either the maintenance of native growth factors during the decellularization process (31), or from the additional immobilization of desired growth factors $(32,33)$. Even though all successful decellularization protocols yield a cell free scaffold many fail to maintain growth factors as in normal livers but most maintain structural proteins. Even though optimal scaffold required for successful recellularization cannot be defined, pre-conditioning of scaffolds and addition of growth factors in culture medium should yield to enhanced implanted cell survival and function.

The dose-dependent induction of proliferation and migration in hFLPC demonstrated that Wnt3a and Wnt5a play an important role in these processes. We found that Wnt3a up-regulated the Wnt target gene Axin-2 and 
DKK2 in hFLPC and increased the proliferation of these cells too. These data are in accordance with the assumption that Axin-2 and DKK2 play a major role in the cell cycle transition $(34,35)$.

The Wnt signalling consists either in a canonical or a non-canonical pathway and so far only the canonical pathway has been better characterized (36) with $\beta$-catenin as the main effector of the canonical signaling. Our results indicate that both canonical and non-canonical pathways of $\mathrm{Wnt} / \beta$-catenin signaling may be involved in proliferation and migration of human liver progenitors. We confirmed by qPCR that Axin-2 and DKK2 expression was substantially elevated by Wnt3a treatment but not by Wnt5a, while CXCR7 was upregulated by Wnt5a only in 1 out of 3 primary hFLPC. Furthermore, blockage of the Wnt pathway in hFLPC was efficiently achieved using both canonical and non-canonical Wnt3a and Wnt5a inhibitors. As a consequence of depressing the expression of these prominent canonical and non-canonical Wnt signaling mediators in hFLPC the migratory and proliferation activity was diminished. On the other hand, studies in animal experiments have reported transforming growth factor- $\alpha$ and cyclin $\mathrm{D}$ as the direct targets of $\beta$-catenin signaling in hepatocyte proliferation (28). It is likely that direct targets of $\mathrm{Wnt} / \beta$-catenin may differ in animal and human livers.

In the liver, Wnt signaling is known to play a dual and paradoxical role. It is engaged in liver cell (I) proliferation both physiologically in regeneration processes and in the oncogenic pathway involved in a subset of liver cancers, (II) as well as physiological signaling as patterning its metabolic zonation in the quiescent liver lobule (37). This dual role has been studied using murine genetic models of $\beta$-catenin, establishing a role of the canonical $W n$ in iner cell proliferation and regeneration (38). All these studies demonstrate an integrated role for Wnt signaling in hepatic liver cell functions.

\section{Conclusions}

We showed for the first time that the Wnt signal transduction pathway is involved in the control of the proliferation and migration of hFLPC. Axin-2, DKK2 and CXCR7 genes are involved in enhanced proliferation of hFLPC. Thus, we propose, localized direct delivery of Wnt3a and Wnt5a to the cells during liver cell transplantation or in vitro regeneration might be beneficial for successful liver regeneration. Future studies should be performed to maintain the structure and function of the hFLPC after a large in vitro expansion and evaluate the long-term outcome of tissue-engineered livers using hFLPC from patient livers.

\section{Acknowledgments}

Funding: This study was financed by the Swedish Government LUA ALF grant to SSH. The funding body never had any role in study design, data collection, analysis, interpretation and writing of manuscript.

\section{Footnote}

Conflicts of Interest: All authors have completed the ICMJE uniform disclosure form (available at http://dx.doi. org/10.21037/tgh.2020.01.12). The authors have no conflicts of interest to declare.

Ethical Statement: The authors are accountable for all aspects of the work in ensuring that questions related to the accuracy or integrity of any part of the work are appropriately investigated and resolved. Use of fetal tissue in the study is in accordance with ethical permission from Regionala etikprövningsnämden i Göteborg (No. 198-11). Written informed consent was obtained from all patients. The study was conducted in accordance with the Declaration of Helsinki (as revised in 2013).

Open Access Statement: This is an Open Access article distributed in accordance with the Creative Commons Attribution-NonCommercial-NoDerivs 4.0 International License (CC BY-NC-ND 4.0), which permits the noncommercial replication and distribution of the article with the strict proviso that no changes or edits are made and the original work is properly cited (including links to both the formal publication through the relevant DOI and the license). See: https://creativecommons.org/licenses/by-nc-nd/4.0/.

\section{References}

1. Lozano R, Naghavi M, Foreman K, et al. Global and regional mortality from 235 causes of death for 20 age groups in 1990 and 2010: a systematic analysis for the Global Burden of Disease Study 2010. Lancet 2012;380:2095-128.

2. Everhart JE, Ruhl CE. Burden of digestive diseases in the United States Part III: Liver, biliary tract, and pancreas. Gastroenterology 2009;136:1134-44. 
3. Murray CJL, Lopez AD. The Global burden of disease : a comprehensive assessment of mortality and disability from diseases, injuries, and risk factors in 1990 and projected to 2020 : summary / edited by Christopher J. L. Murray, Alan D. Lopez. Geneva: World Health Organization, 1996.

4. Fitzpatrick E, Mitry RR, Dhawan A. Human hepatocyte transplantation: state of the art. J Intern Med 2009;266:339-57.

5. Ribes-Koninckx C, Ibars EP, Calzado Agrasot MA, et al. Clinical outcome of hepatocyte transplantation in four pediatric patients with inherited metabolic diseases. Cell Transplant 2012;21:2267-82.

6. Pareja E, Gomez-Lechon MJ, Cortes M, et al. Human hepatocyte transplantation in patients with hepatic failure awaiting a graft. Eur Surg Res 2013;50:273-81.

7. Shirakigawa N, Ijima H, Takei T. Decellularized liver as a practical scaffold with a vascular network template for liver tissue engineering. J Biosci Bioeng 2012;114:546-51.

8. Peloso A, Ferrario J, Maiga B, et al. Creation and implantation of acellular rat renal ECM-based scaffolds. Organogenesis 2015;11:58-74.

9. Mazza G, Rombouts K, Rennie Hall A, et al. Decellularized human liver as a natural 3D-scaffold for liver bioengineering and transplantation. Sci Rep 2015;5:13079.

10. Hussein KH, Park KM, Kim HM, et al. Construction of a biocompatible decellularized porcine hepatic lobe for liver bioengineering. Int J Artif Organs 2015;38:96-104.

11. Navarro-Tableros V, Herrera Sanchez MB, Figliolini F, et al. Recellularization of rat liver scaffolds by human liver stem cells. Tissue Eng Part A 2015;21:1929-39.

12. Hussein KH, Park KM, Kang KS, et al. Heparin-gelatin mixture improves vascular reconstruction efficiency and hepatic function in bioengineered livers. Acta Biomater 2016;38:82-93.

13. Park KM, Hussein KH, Hong SH, et al. Decellularized Liver Extracellular Matrix as Promising Tools for Transplantable Bioengineered Liver Promotes Hepatic Lineage Commitments of Induced Pluripotent Stem Cells. Tissue Eng Part A 2016;22:449-60.

14. Kadota Y, Yagi H, Inomata K, et al. Mesenchymal stem cells support hepatocyte function in engineered liver grafts. Organogenesis 2014;10:268-77.

15. Sekine S, Gutierrez PJ, Lan BY, et al. Liver-specific loss of beta-catenin results in delayed hepatocyte proliferation after partial hepatectomy. Hepatology 2007;45:361-8.

16. Goessling W, North TE, Lord AM, et al. APC mutant zebrafish uncover a changing temporal requirement for wnt signaling in liver development. Dev Biol 2008;320:161-74.

17. Tan X, Behari J, Cieply B, et al. Conditional deletion of beta-catenin reveals its role in liver growth and regeneration. Gastroenterology 2006;131:1561-72.

18. Reya T, Clevers H. Wnt signalling in stem cells and cancer. Nature 2005;434:843-50.

19. Yang W, Yan HX, Chen L, et al. Wnt/beta-catenin signaling contributes to activation of normal and tumorigenic liver progenitor cells. Cancer Res 2008;68:4287-95.

20. Apte U, Thompson MD, Cui S, et al. Wnt/betacatenin signaling mediates oval cell response in rodents. Hepatology 2008;47:288-95.

21. Hu M, Kurobe M, Jeong YJ, et al. Wnt/beta-catenin signaling in murine hepatic transit amplifying progenitor cells. Gastroenterology 2007;133:1579-91.

22. Patil PB, Begum S, Joshi M, et al. Phenotypic and in vivo functional characterization of immortalized human fetal liver cells. Scand J Gastroenterol 2014;49:705-14.

23. Kuhl M, Sheldahl LC, Park M, et al. The Wnt/Ca2+ pathway: a new vertebrate $W$ nt signaling pathway takes shape. Trends Genet 2000;16:279-83.

24. Ishitani T, Kishida S, Hyodo-Miura J, et al. The TAK1NLK mitogen-activated protein kinase cascade functions in the Wnt-5a/Ca(2+) pathway to antagonize Wnt/betacatenin signaling. Mol Cell Biol 2003;23:131-9.

25. Yadav A, Gupta A, Yadav S, et al. Association of Wnt signaling pathway genetic variants in gallbladder cancer susceptibility and survival. Tumour Biol 2016;37:8083-95.

26. Huang Y, Ye Y, Long P, et al. Silencing of CXCR4 and CXCR7 expression by RNA interference suppresses human endometrial carcinoma growth in vivo. Am J Transl Res 2017;9:1896-904.

27. Uygun BE, Soto-Gutierrez A, Yagi H, et al. Organ reengineering through development of a transplantable recellularized liver graft using decellularized liver matrix. Nat Med 2010;16:814-20.

28. Torre C, Benhamouche S, Mitchell C, et al. The transforming growth factor-alpha and cyclin D1 genes are direct targets of beta-catenin signaling in hepatocyte proliferation. J Hepatol 2011;55:86-95.

29. Jain RK, Au P, Tam J, et al. Engineering vascularized tissue. Nat Biotechnol 2005;23:821-3.

30. Martino MM, Briquez PS, Guc E, et al. Growth factors engineered for super-affinity to the extracellular matrix enhance tissue healing. Science 2014;343:885-8.

31. Yagi H, Fukumitsu K, Fukuda K, et al. Human-scale 
whole-organ bioengineering for liver transplantation: a regenerative medicine approach. Cell Transplant 2013;22:231-42.

32. Conklin BS, Wu H, Lin PH, et al. Basic fibroblast growth factor coating and endothelial cell seeding of a decellularized heparin-coated vascular graft. Artif Organs 2004;28:668-75.

33. Nakamura S, Ijima H. Solubilized matrix derived from decellularized liver as a growth factor-immobilizable scaffold for hepatocyte culture. J Biosci Bioeng 2013;116:746-53.

34. Hadjihannas MV, Bernkopf DB, Bruckner M, et al. Cell cycle control of Wnt/beta-catenin signalling by conductin/ axin2 through CDC20. EMBO Rep 2012;13:347-54.

35. Mu J, Hui T, Shao B, et al. Dickkopf-related protein 2 induces G0/G1 arrest and apoptosis through suppressing Wnt/beta-catenin signaling and is frequently methylated in breast cancer. Oncotarget 2017;8:39443-59.

36. Clevers H, Nusse R. Wnt/beta-catenin signaling and disease. Cell 2012;149:1192-205.

37. Gougelet A, Colnot S. A Complex Interplay between Wnt/beta-Catenin Signalling and the Cell Cycle in the Adult Liver. Int J Hepatol 2012;2012:816125.

38. Niehrs C, Acebron SP. Mitotic and mitogenic Wnt signalling. EMBO J 2012;31:2705-13. doi: $10.21037 / \operatorname{tgh} .2020 .01 .12$

Cite this article as: Liu Z, Kuna VK, Xu B, SumitranHolgersson S. Wnt ligands $3 \mathrm{a}$ and $5 \mathrm{a}$ regulate proliferation and migration in human fetal liver progenitor cells. Transl Gastroenterol Hepatol 2021;6:56. 